\title{
Editorial
}

\section{Beobachtungen und Gedanken zur medizinischen Entwicklungszusammenarbeit - Ein Brief aus Kolumbien}

\section{Th. Abelin ${ }^{1}$ und E. Zehnder ${ }^{2}$ zurzeit Cali, Kolumbien}

Sich an der medizinischen Aufbauarbeit in einem Entwicklungsland zu beteiligen, ist der Wunschtraum manchen Arztes, mancher Krankenschwester und Laborantin. Bis vor kurzem bestimmte das Leitbild des väterlichen Urwalddoktors die Vorstellungen, die man sich von der medizinischen Arbeit in der dritten Welt machte - ein idealisiertes Leitbild, in dem ein Arzt die Wunder der modernen Medizin in ein abgelegenes Dorf Afrikas, Südamerikas oder Asiens bringt. Stundenlang warten unzählige, von weit hergekommene Patienten, um vom fremden Medizinmann untersucht und behandelt zu werden, während dieser seinerseits durch die sichtbaren Heilerfolge seiner Tätigkeit belohnt wird.

Diese fand ihren Sinn in erster Linie in der augenfälligen und dankbaren Direkthilfe am leidenden Mitmenschen, während weniger nach dem Stand der Gesundheitsversorgung der Gesamtbevölkerung gefragt wurde. Erst in den frühen sechziger Jahren gewann eine mehr epidemiologisch beeinflusste Betrachtungsweise an Bedeutung: Wie viele Einwohner sind in einer Provinz gesundheitlich zu versorgen? Welches sind die wichtigsten Krankheits- und Todesursachen? Welche materiellen und personellen Mittel (Spitäler, Konsultorien, Ärzte, Krankenschwestern usw.) wären notwendig, um der Gesamtbevölkerung der Gegend eine medizinische Versorgung im konventionellen Sinn zu gewährleisten? Gibt es dazu ein alternatives Gesundheitsversorgungssystem, das die ganze Bevölkerung erreichen kann und welches den ökonomischen Möglichkeiten und soziokulturellen Verhältnissen genügend Rechnung trägt?

Von diesen neuen Fragestellungen ausgehend wurden in der Folge recht verschiedenartige Experimentierprojekte in die Wege geleitet, mit dem Ziel, mit wenigen und einfachen Mitteln der Gesamtbevölkerung einer bestimmten Gegend eine Basisgesundheitsversorgung zu ermöglichen. Als besonders erfolgreich erwiesen sich dabei Modelle, die dem medizinischen Hilfspersonal eine zentrale Rolle zuordneten und das Angebot zur Hauptsache auf die

Korrespondenzadressen:

1 Prof. Dr, med., Institut für Sozial- und Präventivmedizin der Universität Bern, Inselspital, CH-3010 Bern.

2 Dr. med., Apdo, aéreo 20300, Cali, Colombia (bis Oktober 1979).
Behandlung von einfachen, häufigen Krankheiten (v. a. Diarrhoe, Parasiten, Malaria usw.) und präventivmedizinische Massnahmen (Impfaktionen usw.) beschränkten. Unterdessen haben einzelne Länder der Dritten Welt ihre Gesundheitsversorgung in diesem «revolutionären» Sinne umstrukturiert (z. B. China, Tansania). In anderen Gegenden ist die Experimentierphase so weit fortgeschritten, dass man darangehen kann, die gewonnenen Erfahrungen einer breiten Bevölkerung zugänglich zu machen.

Wir haben zurzeit die Möglichkeit, diese faszinierende Entwicklung als Gast-Mitarbeiter des Instituts für Sozial- und Präventivmedizin der Universidad' del Valle in Cali, Kolumbien, aus nächster Nähe mitzuerleben. Cali ist eine typische südamerikanische Grossstadt, deren Bevölkerung in den letzten fünf Jahren um rund $25 \%$ auf 1240000 Einwohner angewachsen ist. Ständig werden neue Stadtteile aus schäbigen Karton- und Blechhütten, anfänglich ohne Anschluss an Stromnetz, Wasserversorgung und Kanalisation, aus dem Boden gestampft. Zugleich ist Cali - und das ist das Besondere an dieser Stadt - seit vielen Jahren ein Experimentierfeld für Fragen der gesundheitlichen Primärversorgung.

Bereits im Jahre 1963 organisierte die medizinische Fakultät der Universidad del Valle mit ausländischer Hilfe in einer Kleinstadt in der Nähe von Cali eine weitgehend auf freiwillige Mitarbeiterinnen gestützte gesundheitliche Versorgung (sog. Projekt Candelaria). In systematischen Erhebungen wurden wertvolle Daten über den Gesundheitszustand der Bevölkerung und die vordringlichen Notwendigkeiten und Bedürfnisse der Bewohner gesammelt. Regelmässige Hausbesuche der sogenannten «Promotorinnen» dienten dazu, das Wachstum der Kinder zu kontrollieren, die gängigen Impfungen zu verabreichen, einfache Behandlungen (z. B. der häufigen Durchfallerkrankung) durchzuführen und den Eltern gesundheitsgerechteres Verhalten zu instruieren. Ein «Gesundheitspass» im Besitz jeder Mutter sollte deren Interesse an der gesundheitlichen Entwicklung ihrer Kleinkinder vergrössern. Medizinstudenten erhielten die Möglichkeit, als Mitarbeiter im Basisspital die im Rahmen der neustrukturierten Primärversorgung veränderte Rolle des Arztes kennenzulernen. 
Im Jahre 1972 wurde das Projekt von Candelaria abgeschlossen. Aufgrund der gewonnenen Erfahrungen wurde in der Folge für einen Stadtteil von Cali mit rund 60000 Einwohnern ein neues, diesmal auf hauptamtliche Promotorinnen gestütztes Versorgungssystem aufgebaut (sog. Projekt Primops). Während die wissenschaftliche Planung und Evaluation von Mitarbeitern des sozialmedizinischen Instituts übernommen wurde, trug die städtische Gesundheitsbehörde die Verantwortung für die praktische Durchführung und Finanzierung.

Ein anderes Versuchsprojekt testet den Einsatz von Promotorinnen unter ländlichen Verhältnissen (sog. Projekt Cimder), wobei die Gesundheitsversorgung nur Teil eines integralen Entwicklungsprogrammes ist. Eine multidisziplinäre Direktion erarbeitet die Konzepte, überwacht die Durchführung und wertet die angewandten Methoden aus. Besonders bemerkenswert sind einige für die medizinische Betreuung der Bevölkerung entwickelte «Instrumente», die sich ausgezeichnet bewährt haben: ein dreifarbiger Messstreifen (grün-orange-rot) zur Feststellung der Unterernährung aufgrund des Oberarmumfangs, ein einfaches Gerät zur Trinkwasserchlorierung, eine einfache «Dorfapotheke» mit sechs Basismedikamenten, ein Kleinlaboratorium mit einfachen Streifentests sowie ein übersichtliches und handliches Karteikartensystem, in dem die Promotorin Daten und erbrachte Leistungen der betreuten Familien registriert.

Pimar ist der Name eines weiteren interessanten Experimentierprojekts, das von zwei Chefärzten von Kleinspitälern für eine Bevölkerung von rund 90000 unterprivilegierte Bewohner von Cali entwickelt worden ist. Medizinische Hilfspersonen führen regelmässige Hausbesuche im Quartier durch und übernehmen wichtige Kontroll- und Triagefunktionen. Das Spital kann sich dadurch gezielter mit ernsthaft Kranken beschäftigen und erfüllt damit erst richtig den ihm zugewiesenen Auftrag. Bemerkenswert ist - unter anderem - der von Pimar gegründete Hypertonikerklub mit rund 400 Mitgliedern, der zur regelmässigen Blutdruckkontrolle und ununterbrochenen Behandlung motiviert und den gemeinsamen Ankauf von verbilligten Medikamenten ermöglicht.

Besonders bemerkenswert fanden wir die Ergebnisse eines psychiatrischen Versuchsprojektes. Eine zufällig ausgelesene Gruppe psychisch kranker Menschen wurde von gut vorbereitetem Hilfspersonal in ihrem angestammten Milieu betreut und behandelt. Diese «Laien»-Mitarbeiter besuchten ihre Patienten täglich zu Hause, halfen bei der Lösung auftretender Probleme mit, besprachen sich mit den Angehörigen und sorgten, sofern nötig, für eine regelmässige Medikamenteneinnahme. Psychiatrische Fachleute übernahmen die regelmässige Supervision. Die erreichten Therapieresultate wur- den mit den Erfolgen des konventionellen Systems (Spezialklinik, Facharzt) verglichen und waren absolut ebenbürtig. Besonders günstig für das neuartige Versorgungsmodell fiel ein Kosten-NutzenVergleich der beiden Systeme aus.

Neuartig und bestechend erschien uns auch die Tätigkeit einer interdisziplinären Expertengruppe (sog. Projekt Prides), die sich darauf spezialisiert hat, bestehende Institutionen des Gesundheitswesens auf ihre organisatorische Zweckmässigkeit und ihre Wirksamkeit zu evaluieren und sinnvolle Reorganisationsvorschläge oder Alternativmodelle im Sinne der wichtigsten Bedürfnisse der Bevölkerung und des rationellen Gebrauchs der zur Verfügung stehenden Mittel zu erarbeiten. Der Gedanke war für uns naheliegend, dass ein Organismus wie Prides auch im schweizerischen Gesundheitswesen wichtige Aufgaben zu erfüllen hätte.

Ein weiteres Projekt (sog. Programa de Nutrición) besteht aus einer Fachgruppe mit 20 Akademikern verschiedenster Richtungen und beschäftigt sich mit allen Aspekten der Ernährung. Basis für ihre Tätigkeit ist ein Modell über die Verursachung der Unterernährung, welches medizinische, soziokulturelle, agronomische und ökonomische Faktoren berücksichtigt. Aufgrund von ausgewählten Indikatoren werden im ganzen Land mit systematischen Stichprobenerhebungen Daten gesammelt, die dem nationalen Planungsministerium zur Verfügung gestellt werden und diesem erlauben sollten, die laufenden Ernährungsprogramme (sog. Plan Pan) zu evaluieren und zu verbessern. Eines der Ergebnisse eines Projekts dieser Gruppe zeigt, dass kurze Stilldauer und frühes Einsetzen mit Zusatznahrung mit häufigem Auftreten von Durchfall und nachfolgenden Symptomen von Unterernährung korreliert sind. Aufgrund dieser Resultate soll nun in Cali eine massive Kampagne zur Förderung der Laktation durchgeführt werden.

Schliesslich sei noch auf das Projekt «Vivamos mejor» hingewiesen, das seit fünf Jahren in Cali funktioniert und bisher vorwiegend aus schweizerischen Quellen finanziert wurde. "Vivamos mejor» hat sich zum Ziel gesetzt, in den Armenvierteln der Stadt gesundheitserzieherische Programme durchzuführen und damit einen Beitrag zur Verbesserung der gesundheitlichen Situation zu leisten. Besonders gut konzipiert ist die Tätigkeit der seit zwei Jahren funktionierenden «Kindergärten» für unterernährte und vernachlässigte Kinder im Vorschulalter. Ziel der jeweils halbjährigen Programme ist es, die Kleinkinder psychisch und physisch $z u$ fördern und gleichzeitig die Mütter in praktischen Kursen zu unterrichten, wie sie mit den ihnen zur Verfügung stehenden einfachen Mitteln Unterernährung vermeiden und ihre Kinder psychomotorisch fördern können. Dieses Modell findet bei staatlichen 
Stellen grosses Interesse und hat gute Chancen, nach Abschluss der laufenden Experimentierphase viel breitere Anwendung zu finden.

Die Vielfältigkeit der beschriebenen Modellprojekte soll nicht darüber hinwegtäuschen, dass für einen beträchtlichen Teil der Bewohner von Cali weiterhin keine funktionstüchtige Gesundheitsversorgung besteht. Die beschriebenen Experimente hätten wenig Sinn, wenn es nicht gelänge, die daraus gewonnenen Erfahrungen der ganzen marginalen Bevölkerung der Stadt zugänglich zu machen. Die Experimentierphase ist heute vorbei, die Zeit der breiten Anwendung ist gekommen. Diese wichtige Entwicklung wurde vor einigen Monaten vom neu ernannten Direktor der städtischen Gesundheitsbehörden (einem von der Universität freigestellten Dozenten für Sozialmedizin) in die Wege geleitet. Zusammen mit verschiedenen Fachleuten der beschriebenen Modellprogramme arbeitet er zurzeit ein Konzept zur Primärversorgung der gesamten Armenviertelbevölkerung von Cali (rund eine halbe Million Menschen) aus und sucht deren Finanzierung zu sichern.

Ausländische Hilfe spielte bei praktisch all den beschriebenen Projekten in kleinerem oder grösserem Masse eine Rolle. Im Projekt Candelaria arbeiteten noch verschiedene amerikanische Fachleute mit, und auch die Betriebskosten wurden zur Hauptsache von aussen bezahlt. Cimder erhält noch heute beträchtliche Unterstützung aus Kanada, doch liegt die Ausführung praktisch vollständig in kolumbianischen Händen. Primops und Prides schliesslich sind von ausländischer Hilfe unabhängig geworden und laufen heute voll zu Lasten der Gesundheitsbehörde. Pimar hat in der ersten Phase Unterstützung zur Einrichtung von Gesundheitsposten und zur besseren Ausrüstung der Kleinspitäler erhalten. Heute wird es voll durch kolumbianische Mittel finanziert.

Die Universität del Valle unterhält seit gut 20 Jahren intensive Kontakte mit amerikanischen Universitäten und Stiftungen. Verschiedene Mitarbeiter erhielten die Möglichkeit, an amerikanischen und englischen Universitäten Postgraduate-Ausbildungen zu erhalten. Heute wird eine Spezialausbildung in öffentlicher Gesundheit auch in Cali selbst angeboten. Für die beschriebenen Projekte hatte das zur Folge, dass schon sehr früh eigene Fachleute die volle Verantwortung übernehmen konnten. Dies ermöglichte eine relativ eigenständige und den gegebenen Verhältnissen besonders gut angepasste Entwicklung der Programme. Die finanzielle Ablösung erfolgte einige Jahre später und verlief schrittweise.

Am Beispiel von Cali lassen sich die einzelnen Elemente und Phasen bestimmen, die ganz allgemein zur Entwicklung einer sinnvollen, den lokalen Ver- hältnissen angepassten und der ganzen Bevölkerung zugänglichen medizinischen Versorgung erforderlich sind. In erster Linie muss durch kompetente Fachleute des Gesundheitswesens ein vernünftiges, breit anwendbares und ökonomisch tragbares Konzept erarbeitet werden. Dieses wird in einzelnen, gut evaluierten Pilotprojekten getestet und laufend verbessert. In einer weiteren Phase müssen die Mittel beschafft und das Personal rekrutiert werden, um das Konzept einer grossen Bevölkerungsgruppe zugänglich zu machen. Während in der Experimentierphase ausländische Mittel und Experten noch eine zentrale Rolle spielen können, muss in der zweiten Phase die Finanzierung und technische Leitung zur Hauptsache aus eigenen Ressourcen sichergestellt werden, um eine volle Integration in die bestehenden Strukturen zu ermöglichen.

Während in Kolumbien - als typischem Vertreter der sogenannten Dritten Welt - ein staatliches Gesundheitswesen mit einem relativ dichten Netz von Spitälern besteht, verschiedene medizinische Fakultäten Fachpersonal ausbilden und nationale Organisationen sich um besonders akute Probleme (Ernährung, Familienplanung, Krebsvorsorge) kümmern, fehlt in den ärmsten Ländern der sogenannten Vierten Welt die medizinische Infrastruktur praktisch vollständig. In der Regel herrscht dort ein dramatischer Mangel an medizinischem Fachpersonal, und es bestehen auch keine Institutionen, um solches auszubilden; weiter fehlt ein leistungsfähiges Material- und Medikamentennachlieferungssystem, und schliesslich ist auch die öffentliche Verantwortung meist völlig ungenügend. Hier ist es von besonders grosser Wichtigkeit, einfachste Lösungen zu suchen und ganz konsequent Prioritäten zu setzen. Einheimische Fachleute müssen in einem jahrelangen Prozess darauf vorbereitet werden, die Verantwortung für die Projekte zu übernehmen. Ärzte benötigen dazu meist eine Zusatzausbildung.

Die Erfahrungen früherer Jahrzehnte haben deutlich gemacht, wie problematisch es ist, diese Spezialausbildung in Nordamerika, Europa oder Russland anzubieten. Die Absolventen der Kurse kommen dabei mit einer Lebensweise und Kultur in Kontakt, die es ihnen praktisch unmöglich macht, sich weiterhin für die Lösung der Probleme in ihrem Herkunftsland einzusetzen. Bedeutend bessere Erfahrungen macht man, wenn Vertreter der vierten Welt in bestehenden Ausbildungszentren der dritten Welt eine sinnvolle Vorbereitung auf die vorgesehene Tätigkeit im eigenen Land erhalten können. Solche Ausbildungszusammenarbeit zwischen Ländern der Dritten und Vierten Welt verdient eine gezielte Unterstützung durch Entwicklungsorganisationen der industrialisierten Welt.

Mit Genugtuung kann festgestellt werden, dass die in den letzten Jahren gewonnenen Erfahrungen und 
Erkenntnisse über die gesundheitliche Basisversorgung und ihre infrastrukturellen Voraussetzungen seit einiger Zeit auch die Richtung der medizinischen Entwicklungszusammenarbeit der Direktion für Entwicklungszusammenarbeit und humanitäre Hilfe (DEH) des Eidgenössischen Politischen Departements und der grossen privaten Hilfsorganisationen in der Schweiz bestimmen. Es besteht heute kein Zweifel mehr darüber, dass medizinische Entwicklungshilfe eine völlige Abkehr von der bei uns geltenden Form der medizinischen Versorgung erfordert. Auch ist es - wenigstens im medizinischen Bereich - nicht mehr denkbar, dass Entwicklungszusammenarbeit in den Dienst eines materiellen oder anderen Profits für die Schweiz gestellt werden kann. Zum Beispiel ist ein massiver Einsatz junger Schweizer Ärzte in Entwicklungsländern als
Mittel zur Linderung des bei uns herrschenden Úberflusses an Assistenzärzten (wie er hie und da postuliert wird) im Rahmen des modernen Konzepts der gesundheitlichen Primärversorgung undenkbar. Anderseits wird ein gezielter Einsatz sozial- und präventivmedizinisch interessierter und gut vorbereiteter Mediziner bei der Verwirklichung dieses Konzepts in den am wenigsten entwickelten Regionen der Welt noch lange von grossem Nutzen sein. Zum bestmöglichen Einsatz der sich der Schweiz auf diesem Gebiet stellenden Möglichkeiten schiene es uns angebracht, den Kontakt und den Erfahrungsaustausch unter Ärzten und Organisationen, deren Interessen und Erfahrungen im Bereich der medizinischen Basisversorgung in der unterentwickelten Welt liegen, ganz besonders zu fördern.

\title{
Editorial
}

\section{Observations et réflexions sur la coopération au développement dans le domaine de la santé - Lettre de Colombie}

\author{
Th. Abelin ${ }^{1}$ et E. Zehnder ${ }^{2}$, actuellement à Cali, Colombie
}

Participer activement à la mise en place d'une infrastructure sanitaire dans un pays en voie de développement est le rêve de plus d'un médecin, d'une infirmière ou d'une laborantine. II y a peu de temps encore, l'idée que l'on se faisait du travail médical dans le tiers monde était celle du médecin «patriarche» travaillant en brousse ou en forêt, image idéalisée qui le montrait portant dans les villages lointains de l'Afrique, de l'Amérique du Sud ou de l'Asie les miracles de la médecine moderne. Pendant de longues heures, des malades innombrables qui s'étaient déplacés de loin attendaient d'être examinés et soignés par ce docteur venu de l'étranger, qui voyait son activité récompensée par des guérisons spectaculaires.

Cette activité trouvait sa justification dans l'aide directe apportée à des êtres humains souffrants, alors qu'on se posait moins de questions en ce qui

Adresses pour correspondance:

1 Prof. Dr méd., Institut de médecine sociale et préventive de l'Université de Berne, Hôpital de l' lle, CH-3010 Berne.

2 Dr méd., Apdo aéreo 20 300, Cali, Colombie (jusqu'en octobre 1979). concerne une structure sanitaire profitant à l'ensemble de la population. Ce n'est que vers le début des années soixante que des soucis d'ordre plus épidémiologique gagnèrent en importance: Combien d'habitants nécessitent des soins médicaux dans la province? Quelles sont les causes principales de morbidité et de mortalité? Quels sont les moyens en personnel et en matériel nécessaires pour garantir des soins, au sens conventionnel, à l'ensemble d'une région? Existe-t-il un système de soins, une modalité alternative, susceptible d'être étendu à toute la population et tenant suffisamment compte de sa situation économique et socio-culturelle?

Partant de ces interrogations, des projets expérimentaux très divers ont été entrepris. Ce sont principalement les modèles attribuant un rôle primordial au personnel auxiliaire et limitant l'offre à l'essentiel (traitement des maladies fréquentes - diarrhées, parasites, paludisme, etc. - et mesures de médecine sociale et préventive - vaccinations, assainissement, etc.) qui se sont révélés les plus efficaces. Depuis lors, certains pays du tiers monde (la Chine et la Tanzanie, par exemple) ont restructuré leur système sanitaire dans ce sens «révolutionnaire». 